\title{
Survey of physician knowledge about hemochromatosis
}

\author{
Ronald T. Acton, $P h D^{1}$, James C. Barton, $M D^{2}$, Linda Casebeer, $P h D^{3}$, and Lynya Talley, $P h D^{4}$
}

\begin{abstract}
Purpose: To survey physicians about knowledge of hemochromatosis. Methods: A questionnaire was faxed to American physicians. Results: A total of 2,563 evaluable responses were obtained. There were $\geq 70 \%$ correct responses about at-risk population, associated abnormalities, and population screening. There were $32 \%$ and $53 \%$ correct answers about diagnosis and treatment, respectively. A total of $8.0 \%$ and $4.9 \%$ reported asking $>75 \%$ of patients about family history of hemochromatosis and iron overload, respectively. Less than $25 \%$ requested $H F E$ mutation analysis in the previous year. Correct answers were associated with academic practice, internal medicine specialty, and medical school graduation within 10 years. Conclusion: Many physicians have inadequate knowledge about hemochromatosis diagnosis and treatment. Genet Med 2002:4(3):136-141.
\end{abstract}

Key Words: hemochromatosis, iron overload, survey, genetic education, genetic disorders

Hemochromatosis, an autosomal recessive disorder, affects approximately 0.002 to 0.005 of Caucasians of Western European descent and their descendants. ${ }^{1-4}$ In many persons with hemochromatosis, increased absorption of iron from food and drink results in iron overload. If untreated, iron overload can cause hepatic cirrhosis, primary liver cancer, arthropathy, diabetes mellitus, hypogonadism, cardiomyopathy, and reduced longevity. ${ }^{5-8}$ With early diagnosis and treatment, these complications can be prevented.7,9 Nonetheless, many physicians report that they have not diagnosed or treated persons with hemochromatosis, ${ }^{8}$ and patients with hemochromatosis commonly report delayed diagnosis and treatment of their iron overload and associated disorders. ${ }^{10}$

The rapid increase in understanding of the molecular basis of heritable disorders and availability of genetic testing has created complex issues and opportunities regarding the appropriate application of genetic testing, interpretation of test results, and implications for preventive or corrective management interventions. ${ }^{11}$ Thus it is important that health care providers become familiar with the general benefits and limitations of genetic testing in medical practice and make informed decisions about diagnosis and treatment of heritable disorders. ${ }^{12,13}$ However, several studies have revealed that many physicians are not well informed about heritable disorders, believe that information about genetics and disease is

\footnotetext{
From the ${ }^{1}$ Immunogenetics Program, Departments of Microbiology, Medicine, and Epidemiology, University of Alabama at Birmingham, Alabama; ${ }^{2}$ Division of Hematology/Oncology, Department of Medicine, University of Alabama at Birmingham, Alabama, and Southern Iron Disorders Center, Birmingham, Alabama; ${ }^{3}$ Continuing Medical Education, School of Medicine, University of Alabama at Birmingham, Alabama; ${ }^{4}$ Medical Statistics Section, Department of Medicine, University of Alabama at Birmingham, Alabama.

Ronald T. Acton, PhD, Immunogenetics Program, Room 420, Kaul Genetics Building, 1530 3rd Avenue South, Birmingham, AL 35294-0021.

Received: January 2, 2002
}

Accepted: March 1, 2002. irrelevant to their practices, or lack confidence in using genetic information to manage their patients. ${ }^{12-18}$

There have been few attempts to increase physician knowledge about the role of genetics in the diagnosis and treatment of hemochromatosis and associated iron overload. In one study, primary care, internal medicine, and medicine subspecialty physicians diagnosed greater numbers of hemochromatosis probands after they participated in an education program about hemochromatosis. ${ }^{8}$ However, there is a paucity of reported information about physician knowledge of hemochromatosis and iron overload. Herein, we summarize the responses of 2,563 physicians in the United States to a questionnaire survey about hemochromatosis. The potential applicability of the present results in formulating education programs to increase the diagnosis and treatment of hemochromatosis and iron overload is also discussed.

\section{MATERIALS AND METHODS}

\section{Survey development}

A questionnaire survey of physicians was developed; a copy of the survey is available from the authors upon request. Because this is the first survey of its kind, there was no available a priori information to guide our strategy to evaluate physician knowledge of hemochromatosis. Accordingly, we chose to identify fundamental clinical knowledge questions from a Practice Parameter for Hereditary Hemochromatosis of the College of American Pathologists ${ }^{7}$ and The Hemochromatosis Management Working Group of the Centers of Disease Control and Prevention. ${ }^{9}$ The purposes of the survey included assessment of (1) physician and practice demographic characteristics (gender, education, type of medical practice, practice location, and racial/ethnic composition of practice); (2) physician knowledge about hemochromatosis epidemiology (Questions 1, 2), genetics (Questions 3, 4), pathogenesis 
(Questions 6, 7, and 8), diagnosis (Questions 5, 10), treatment (Question 9), and screening (Question 11); (3) frequencies with which physicians obtain family history of disease pertinent to hemochromatosis- and iron overload-associated disorders (hemochromatosis, iron overload, diabetes mellitus, and hepatic cirrhosis); (4) racial/ethnic group composition of physicians' practices; (5) frequency of diagnosis and treatment of hemochromatosis and associated iron overload and of HFE mutation analysis in physicians' practices; and (6) methods by which physicians report that they prefer to obtain new medical and continuing education information. Five physicians representing family practice, general practice, internal medicine, and hematology reviewed the survey for content, clarity, and estimated time to complete the survey $(15 \mathrm{~min})$. The survey and study was approved by the Institutional Review Board of the University of Alabama at Birmingham. In the introduction section of the survey, this statement was included, "Your completion of the survey implies your consent to participate in this research project.”

\section{Survey participants}

We transmitted questionnaires to family practice, general practice, obstetrics/gynecology, general internal medicine, and internal medicine subspecialty (gastroenterology, hematology, and medical oncology) physicians, because they diagnose most cases of hemochromatosis in routine medical care delivery. 7,8 From computerized lists of the American Medical Association (AMA), 142,318 physicians in these categories were identified. A statistical power calculation revealed that a sample size of 2,500 responders with evaluable questionnaires would constitute a representative sample. A random sample, not further stratified by specialty, was faxed from the total sample using the AMA physician lists. We began transmitting our survey in January of 2000 and continued until more than 2,500 evaluable responses were received. Each responder received 1 hour of AMA Category I continuing medical education credit.

\section{Statistical analysis}

A preliminary power calculation was conducted using a two-sided alpha $=0.05$ with power $=80 \%$. Thus we determined that 2,500 responses would be needed to generalize the overall population of these specialists within the United States. A random stratified sample pool was created and surveys were faxed from this pool until we received the required number of $>2,500$ responses. Descriptive statistics were expressed as enumerations, percentages, means ( $\pm 1 \mathrm{SD}$ ), and ranges. The Cochran-Mantel-Haenszel chi-square test was used to evaluate associations between various factors and correctly answering all questions in a clinical knowledge area. ${ }^{19}$ Fisher's exact test was used when expected cell frequencies were less than five. ${ }^{20}$ Multivariate logistic regression analyses were performed using a backward elimination procedure to identify significant predictors for answering all questions correctly within clinical knowledge areas. Variables included in all models before the backward elimination procedure included gender, specialty, years since graduation from medical school, type of practice, location of practice, and Internet access. Because so few persons answered all 11 hemochromatosis and iron overload knowledge questions correctly $(N=29)$, answering questions correctly was categorized according to the median number of knowledge questions answered correctly (median $=7$ ). Therefore, physicians were categorized as those who answered seven or more questions correctly and those who answered fewer than seven questions correctly. The odds ratio (OR) and 95\% confidence interval (CI) were computed to use as measures of association. Model fit was assessed using the Hosmer-Lemeshow goodness of fit statistic. ${ }^{21}$

\section{RESULTS}

\section{Demographic characteristics of responders and their practices}

We transmitted 8,000 questionnaires and received evaluable responses from 2,563 physicians (overall response rate $32.0 \%$ ). Demographic characteristics of responders are displayed in Table 1 . More than $81 \%$ were men, $38.9 \%$ were family or general practitioners, $83.1 \%$ practiced in solo or group settings, $64.5 \%$ practiced in urban or suburban locations, and approximately three quarters had graduated from medical school $>10$ years before completing the survey. A total of $47.9 \%$ of responders reported that more than $75 \%$ of their patients were Caucasians, and that $1.4 \%$ and $0.9 \%$ reported that more than $75 \%$ of their patients were Hispanics and African Americans, respectively. Only $0.6 \%$ reported that more than $75 \%$ of their patients were Asians.

\section{Knowledge about hemochromatosis and associated iron overload}

A total of $77.7 \%$ of responders correctly identified Western Europeans as the population group in which hemochromatosis primarily occurs, and that $45.2 \%$ indicated correctly that the approximate frequency of hemochromatosis in populations of Western Europeans and their descendants is 1 in 200. Most $(78.7 \%)$ responders correctly indicated that males and females at all stages of life can have hemochromatosis, and $71.8 \%$ identified the gene responsible for "classical" hemochromatosis and its pattern of inheritance. Characteristics of persons with hemochromatosis with relatively severe iron overload were correctly identified by $87.4 \%$ of responders. However, only $12.6 \%$ correctly indicated that none of the indicators of severe iron overload were necessarily diagnostic of hemochromatosis. Only $31.5 \%$ indicated that hemochromatosis can be detected before iron overload occurs by demonstration of elevated saturation of transferrin with iron, whereas $85.1 \%$ identified typical complications of iron overload in hemochromatosis. However, $63.9 \%$ identified the consequence of inheriting two HFE mutations. Multisystem consequences of iron-induced injury were recognized by $85.1 \%$ of responders. Therapeutic phlebotomy was recognized as the preferred treatment for iron overload due to hemochromatosis by $53.3 \%$ of responders. The rationale and potential advantages of population screening for hemochromatosis were selected by $83.9 \%$ of responders. Overall, $7.9 \%$ of responders answered $90 \%$ to $100 \%$ of the questions correctly, $12.8 \%$ an- 
Table 1

Demographic and practice characteristics of 2,563 physician responders

\begin{tabular}{|c|c|}
\hline Characteristic & $\%$ \\
\hline \multicolumn{2}{|l|}{ Gender } \\
\hline Men & 81.3 \\
\hline Women & 18.7 \\
\hline \multicolumn{2}{|l|}{ Specialty } \\
\hline Family practice & 34.2 \\
\hline General practice & 4.7 \\
\hline General internal medicine and subspecialties & 32.1 \\
\hline Other $^{a}$ & 16.4 \\
\hline Unstated & 12.6 \\
\hline \multicolumn{2}{|l|}{ Type of practice } \\
\hline Solo & 44.1 \\
\hline Group & 39.0 \\
\hline Academic & 4.6 \\
\hline HMO & 0.5 \\
\hline Other $^{a}$ & 2.6 \\
\hline Unstated & 9.2 \\
\hline \multicolumn{2}{|l|}{ Practice location } \\
\hline Urban & 28.1 \\
\hline Suburban & 36.3 \\
\hline Rural & 26.7 \\
\hline Unstated & 8.9 \\
\hline \multicolumn{2}{|l|}{ Years since graduation from medical school } \\
\hline$\leq 10$ & 18.0 \\
\hline $11-20$ & 32.3 \\
\hline$>20$ & 41.7 \\
\hline Unstated & 8.0 \\
\hline
\end{tabular}

${ }^{a}$ The present survey was faxed to physicians known to the AMA as family practice, general practice, obstetrics/gynecology, general internal medicine, and internal medicine subspecialty (gastroenterology, hematology, and medical oncology). However, 31 other physician specialty categories were identified by certain respective responders, including obstetrics/gynecology. Because there were insufficient numbers of responders in any of these 31 categories for separate analysis, these data were combined under the present "Other" category.

swered $80 \%$ to $89 \%$ of the questions correctly, and $16.5 \%$ answered $70 \%$ to $79 \%$ of the questions correctly. Percentages of physicians who answered questions correctly are stratified by specialty and type of practice in Table 2 .

\section{Obtaining family history of disease}

Only $8.0 \%$ of responders reported that they asked more than $75 \%$ of their patients about their family history of hemochromatosis, and $4.9 \%$ asked more than $75 \%$ of their patients about a possible family history of iron overload. In contrast, $79.9 \%$ reported that they asked more than $75 \%$ of their patients about their family history of diabetes mellitus, and $21.8 \%$ asked more
Table 2

Percentages of physicians who answered survey questions correctly

\begin{tabular}{|c|c|c|c|c|}
\hline Characteristic & $90-100 \%$ & $80-89 \%$ & $70-79 \%$ & $<70 \%$ \\
\hline \multicolumn{5}{|l|}{ Specialty } \\
\hline Family practice & 3.9 & 8.1 & 15.6 & 72.4 \\
\hline General practice & 3.3 & 3.3 & 15.7 & 77.7 \\
\hline Internal medicine & 11.7 & 16.7 & 20.2 & 52.5 \\
\hline Other $^{a}$ & 12.4 & 17.9 & 13.3 & 56.9 \\
\hline Unknown & 7.5 & 13.7 & 14.0 & 64.9 \\
\hline \multicolumn{5}{|l|}{ Type of practice } \\
\hline Solo & 4.7 & 9.4 & 15.3 & 70.1 \\
\hline Group & 10.2 & 15.7 & 17.6 & 56.5 \\
\hline Academic & 22.2 & 23.9 & 16.2 & 37.6 \\
\hline $\mathrm{HMO}$ & 7.1 & 35.7 & 0 & 57.1 \\
\hline Other $^{a}$ & 10.6 & 10.6 & 19.7 & 59.1 \\
\hline Unknown & 5.5 & 11.0 & 17.8 & 65.7 \\
\hline
\end{tabular}

${ }^{a}$ The present survey was faxed to physicians known to the AMA as family practice, general practice, obstetrics/gynecology, general internal medicine, and internal medicine subspecialty (gastroenterology, hematology, and medical oncology). However, 31 other physician specialty categories were identified by certain respective responders, including obstetrics/gynecology. Because there were insufficient numbers of responders in any of these 31 categories for separate analysis, these data were combined under the present "Other" category.

than $75 \%$ of their patients about their family history of hepatic cirrhosis.

\section{Frequency of hemochromatosis diagnosis and treatment and HFE mutation analysis in physicians' practices}

In the year preceding their response to the present survey, $56.9 \%$ of responders reported that there had been no new cases of hemochromatosis diagnosed in their practices. A total of $38.5 \%$ of responders reported that there had been one, two, or three new cases diagnosed in their practices, and $4.6 \%$ reported that there had been four or more cases diagnosed in their practices. In addition, $75.6 \%$ of responders reported that they had not referred patients in their practices for HFE mutation analysis in the preceding year; however, $16.8 \%$ reported that they had referred one, two, or three patients for HFE mutation analysis in the preceding year, and $7.6 \%$ had referred four or more patients. Over one quarter (26.3\%) of responders indicated that hemochromatosis was "extremely rare" or "nonexistent" in their practices.

Location of practice was the only physician characteristic significantly associated with patient referral for HFE mutation testing. Physicians who reported having an urban or suburban practice were more likely than physicians who reported having a rural practice to refer their patients for testing $(\mathrm{OR}=1.61$; $95 \%$ CI, 1.26-2.06). Physician specialty was the only physician characteristic significantly associated with diagnosing one or more new cases of hemochromatosis per year. Physicians who reported their specialty as family practice were $50 \%$ less likely 
to diagnose one or more new cases than those physicians who reported their practice as internal medicine $(\mathrm{OR}=0.5 ; 95 \%$ CI, $0.38-0.65)$. Physicians who reported their specialty as general practice were $76 \%$ less likely to diagnose one or more new cases of hemochromatosis than those physicians who reported their specialty as internal medicine $(\mathrm{OR}=0.24 ; 95 \% \mathrm{CI}, 0.10$ 0.57 ). Whereas $71.8 \%$ of responders correctly answered a question about the association of inheriting common HFE mutations, only $24.4 \%$ of responders reported that they had referred patients for HFE testing in the preceding year.

\section{Physician characteristics that were significant predictors of answering questions correctly}

Specialty, years since graduation from medical school, type of practice, and Internet access were significant predictors for answering seven or more general knowledge questions about hemochromatosis correctly (Table 3). We also identified predictors of correct responses to questions about specific topics. Physicians in an academic setting were more likely to answer both epidemiology questions correctly than physicians who were in a solo or group setting (OR $=2.47$; 95\% CI, $1.64-$ 3.72). Family practice and general practice physicians were $28 \%$ less likely than physicians who classified their specialty as "other" to answer the two epidemiology questions correctly $(\mathrm{OR}=0.72 ; 95 \% \mathrm{CI}, 0.56-0.93)$.

Physicians in an academic setting were more likely to answer all genetics questions correctly than physicians in a solo or

\section{Table 3}

Significant predictors of answering questions correctly among 2,563 physician responders

\begin{tabular}{lcc}
\hline Physician characteristics & Odds ratio & $\begin{array}{c}95 \% \text { Confidence } \\
\text { interval }\end{array}$ \\
\hline Specialty & 0.5 & $0.38-0.58$ \\
$\quad$ Family practice & 0.4 & $0.23-0.57$ \\
$\quad$ General practice & 1.0 & - \\
$\quad$ Internal medicine & & \\
Years since medical school graduation & 0.93 & $0.72-1.21$ \\
$>20$ & 0.70 & $0.54-0.90$ \\
$11-20$ & 1.0 & - \\
$\leq 10$ & & $0.27-0.61$ \\
Type of practice & 0.5 & $0.50-1.14$ \\
Solo & 0.8 & - \\
Group & 1.0 & - \\
Academic & & $1.05-1.62$ \\
Internet access & 1.3 & - \\
Yes & 1.0 & \\
No & &
\end{tabular}

Answering questions correctly was categorized as answering seven or more questions about hemochromatosis epidemiology, genetics, pathogenesis, diagnosis and treatment, and screening correctly versus answering fewer than seven of these questions correctly. group setting ( $\mathrm{OR}=2.5 ; 95 \% \mathrm{CI}, 1.52-4.00)$. Family practice and general practice physicians were less likely than physicians who classified their specialty as internal medicine to answer both genetics questions correctly (OR $=0.8$; 95\% CI $0.65-$ 0.95 ; OR $=0.5 ; 95 \%$ CI, 0.34-0.79). Responders who had graduated from medical school $>20$ years before the survey were less likely to answer both genetics questions correctly than those whose graduation was 0 to 10 years before the survey was conducted $(\mathrm{OR}=0.7 ; 95 \% \mathrm{CI}, 0.54-0.89)$.

Family practice and general practice physicians were less likely than physicians who classified their specialty as internal medicine to answer all pathogenesis questions correctly $(\mathrm{OR}=$ 0.5 ; 95\% CI, $0.35-0.52$; OR $=0.6$; 95\% CI, $0.42-1.00$, respectively). Physicians who listed their practice type as solo or group were less likely than those practicing in an academic setting to answer all pathogenesis questions correctly $(\mathrm{OR}=$ 0.4 ; $95 \%$ CI, $0.29-0.63$; OR $=0.8$; 95\% CI, 0.56-1.19, respectively).

There were no significant predictors observed for physicians answering the diagnosis questions correctly. Physicians in an academic setting were more likely than physicians in a solo or group setting to answer the treatment questions correctly (OR $=2.12$; $95 \% \mathrm{CI}, 1.36-3.30$ ). Family practice and general practice physicians were less likely than physicians who classified their specialty as internal medicine to answer the treatment question correctly $(\mathrm{OR}=0.6 ; 95 \% \mathrm{CI}, 0.49-0.73 ; \mathrm{OR}=0.4$; $95 \%$ CI, $0.24-0.58$, respectively). Physicians in a rural practice were less likely to answer the treatment question correctly than those in an urban or suburban practice $(\mathrm{OR}=0.8 ; 95 \% \mathrm{CI}$, $0.64-0.97)$. Men were more likely than women to answer the treatment question correctly (OR $=1.4 ; 95 \% \mathrm{CI}, 1.10-1.79)$. Responders who had graduated from medical school $>20$ years before the survey were less likely than those whose graduation was 0 to 10 years before the present survey to answer the treatment question correctly ( $\mathrm{OR}=0.7 ; 95 \% \mathrm{CI}, 0.53-0.88)$.

Specialty and Internet access were significant predictors for answering the screening question correctly. Family practice and general practice physicians were less likely than physicians who classified their specialty as internal medicine to answer the screening question correctly $(\mathrm{OR}=0.7 ; 95 \% \mathrm{CI}, 0.52-0.88$; $\mathrm{OR}=0.5 ; 95 \% \mathrm{CI}, 0.27-0.74$, respectively). Physicians with Internet access were more likely than physicians without access to answer the screening question correctly $(\mathrm{OR}=1.4 ; 95 \% \mathrm{CI}$, $1.04-1.76$ ). Of the 1,726 respondents who answered the question about having Internet access, $83.7 \%$ reported that they had Internet access. However, only $8.2 \%$ of responders reported that they used on-line courses as a source of information about genetic disorders.

\section{Obtaining new and continuing medical education information}

The three methods for obtaining updated information on genetic disorders preferred by responders at the time of this survey were journals ( $77.1 \%$ of responders), local continuing medical education meetings (39.4\% of responders), and national meetings ( $35.0 \%$ of responders). For future updates on genetic disorders, $52.8 \%$ of responders reported that they 
would prefer journals, $36.9 \%$ would prefer local continuing medical education meetings, and $33.2 \%$ would prefer continuing medical education by FAX.

\section{DISCUSSION}

This survey reveals that $23 \%$ of physician responders were unaware of the population at risk for developing hemochromatosis, the pathogenesis of iron overload-associated complications, and the potential benefits of screening appropriate populations. Responders were even less knowledgeable about the frequency of hemochromatosis in the Western European populations, the phenotypic criterion for diagnosing hemochromatosis before iron overload occurs, the diagnostic indicators of hemochromatosis, and the preferred treatment of iron overload, on the average. More than one quarter of responders indicated that hemochromatosis was "extremely rare" or "nonexistent" in their practices. Although more than $71 \%$ of responders correctly identified the inheritance pattern of hemochromatosis, $<65 \%$ recognized the consequences of inheriting two hemochromatosis-associated HFE mutations. Taken together, these results indicate that education programs for physicians should emphasize that hemochromatosis is common, that elevated values of serum transferrin saturation can be used to diagnose most cases before iron overload occurs, and that phlebotomy to achieve and maintain normal body stores of iron is the preferred treatment.

The sample we obtained was provided by volunteers, and we are unaware of any reason to suspect that physicians of any particular specialty would be more or less likely to respond than those of another specialty. Substantial bias could occur only if nonresponders answered questions differently than responders. There is the possibility that we obtained a biased sample of physicians due to the availability of continuing medical education credit. However, evaluating this possibility was beyond the scope of the present study. We have no way of determining why physicians did or did not respond, but this is characteristic of any voluntary sample. However, the present overall response rate of $32.0 \%$ is similar to that in other questionnaire surveys of more than 1,000 physicians about their knowledge of genetics and adult-onset genetic disorders (34.0\% and $22.1 \%$, respectively). ${ }^{15,22}$ Providing financial incentives to physicians significantly increases their response rates to questionnaires on knowledge of genetics. ${ }^{23}$

Many physicians may not understand or request HFE mutation analysis to diagnose hemochromatosis. In the present study, $72 \%$ of responders correctly answered a question about genetic features of hemochromatosis, including the importance of HFE mutations, but only $24 \%$ of physicians reported that they had referred at least one patient for HFE mutation analysis in the previous year. A survey of physicians in Arkansas indicated that $21 \%$ of responders were aware of a test to detect HFE mutations, but only $3 \%$ had ordered the test. ${ }^{17}$ The difference in the results of the two studies could be explained by either the opportunity for more physicians to become acquainted with HFE testing during the 14 months between the two surveys, or the greater knowledge of physicians nationwide about HFE testing than those in Arkansas. However, the results of both studies suggest that many physicians do not use genetic testing to diagnose hemochromatosis in suspected probands or evaluate their family members.

Many physicians may not identify family members at risk for hemochromatosis because they do not include hemochromatosis and its complications in their family history inquiries. Eighty percent of the present responders reported that they ask more than three quarters of their patients about a family history of diabetes mellitus. In contrast, only $5 \%$ to $21 \%$ of responders ask more than three quarters their patients about the occurrence of hemochromatosis, iron overload, or hepatic cirrhosis in their families. These data could be interpreted as an indication that physicians believe, in general, that hemochromatosis is rare or that there is a greater contribution of genetic factors to the etiology of diabetes mellitus than to the occurrence of hemochromatosis. This finding is striking because the gene mutations responsible for most cases of hemochromatosis have been defined, are common, and a genetic test is available to detect them, whereas the inheritance pattern(s) of diabetes mellitus is (are) not well defined and the causative mutation(s) are unknown. However, the failure to obtain a detailed family history of hemochromatosis, iron overload, and hepatic cirrhosis reported by many responders to the present survey is consistent with observations from physician surveys about cancer. ${ }^{18,22}$

In the present study, physicians who practiced in academic settings and those who identified their specialty as internal medicine were significantly more likely to answer questions correctly about hemochromatosis epidemiology, genetics, pathogenesis, diagnosis and treatment, and screening than physicians in other specialties. In contrast, other investigators have reported that a primary care specialty practice in which exposure to genetic problems is likely to occur is a significant predictor of knowledge about genetics and genetic tests. ${ }^{14}$ However, approximately two thirds of hemochromatosis probands in a suburban community hospital setting were diagnosed by primary care physicians who had participated in a hemochromatosis education program. ${ }^{8}$ Other significant predictors of knowledge of genetics and genetic tests reported previously include recent graduation from medical school, ${ }^{14,22}$ physician confidence in explaining genetic tests results, and tailoring recommendations for screening based on genetic test results. ${ }^{22}$ Thus the present findings confirm and extend observations regarding the importance of recent medical education in understanding and applying genetic principles to the diagnosis and treatment of heritable disorders.

A majority of physicians who responded to our survey selected journals as a major source of updated information about genetic disorders. Medical journals were also the most frequently cited source of new medical practice information in a survey conducted in 1993. ${ }^{14}$ However, one investigator reported in 1998 that family practice and internal medicine journals had the lowest contents of human and/or medical genetics information. ${ }^{24}$ Thus family practice and internal medicine 
journals may not be as useful presently as other journals or educational formats in providing information that will help their readerships remain knowledgeable about medical genetics.

There are several strategies to educate physicians about hemochromatosis which have been tested or planned in the United States, or which may be applicable to such strategies. A hemochromatosis and iron-overload educational program for health care providers (slide and lecture presentations, personal discussions, and educational materials) and the general public (interviews, appearances and presentations for newspapers, public radio television, community information programs, and patient support groups) was effective in increasing diagnosis of hemochromatosis probands in a suburban community hospital. ${ }^{8}$ Similarly, academic detailing was effective in delivering information to physicians and changing their behavior. ${ }^{25,26}$ Although effective, the success of both of these approaches depends critically on individuals who are devoted to the education strategy. A current, broader approach to education by the Centers for Disease Control and Prevention is the development of brochures appropriate for physicians and the general public, respectively, that will permit wide dissemination of information about hemochromatosis and iron overload (personal communication J.C. Barton). In the present study, we did not collect data which allowed us to formulate effective means to change physician knowledge and practice patterns. Recommendations of this nature, based on physician education and consideration of the role of consumers, are reported for other genetic disorders, ${ }^{25-28}$ and it seems likely that they are also applicable to hemochromatosis education. ${ }^{8}$ Altogether, using a variety of strategies to educate physicians should increase early diagnoses of hemochromatosis, improve management, and reduce morbidity and mortality from this common inherited disorder. $6,7,9$

\section{Acknowledgments}

This study was sponsored in part by a supplement grant to Comprehensive Cancer Center Core Support Grant 5P30 CA 13148 from the National Cancer Institute, National Institutes of Health, from UAB's Continuing Medical Education, and from Southern Iron Disorders Center.

\section{References}

1. Edwards CQ, Griffen LM, Goldgar DE, Skolnick MH, Kushner JP. Prevalence of hemochromatosis among 11,065 presumably healthy blood donors. N Engl J Med 1988;318:1355-1362.

2. McLaren CE, Gordeuk VR, Looker AC, Hasselblad V, Edwards CQ, Griffen LM, Kushner JP, Brittenham GM. Prevalence of heterozygotes for hemochromatosis in the white population of the United States. Blood 1995;86:2021-2027.
3. Smith BN, Kantrowitz W, Grace ND, Greenberg MS, Patton TJ, Ookubo R, Sorger K, Semeraro JG, Doyle JR, Cooper AG, Kamat BR, Maregni LM, Rand WM. Prevalence of hereditary hemochromatosis in a Massachusetts corporation: is Celtic origin a risk factor? Hepatology 1997;25:1439-1446.

4. Phatak PD, Sham RL, Raubertas RF, Dunnigan K, O'Leary MT, Braggins C, Cappuccio JD. Prevalence of hereditary hemochromatosis in 16031 primary care patients. Ann Intern Med 1998;129:954-961.

5. Cartwright GE, Edwards CQ, Kravitz K, Skolnick M, Amos DB, Johnson A, Buskjaer L. Hereditary hemochromatosis: phenotypic expression of the disease. N Engl J Med 1979;301:175-179.

6. Niederau C, Strohmeyer G. Survival in hemochromatosis. In: Barton JC, Edwards CQ, editors. Hemochromatosis: genetics, pathogenesis, diagnosis, and treatment. Cambridge: Cambridge University Press, 2000:359-368.

7. Witte DL, Crosby WH, Edwards CQ, Fairbanks VF, Mitros FA. Hereditary hemochromatosis. Clin Chim Acta 1996;245:139-200.

8. Barton JC, Barton NH, Alford TJ. Diagnosis of hemochromatosis probands in a community hospital. Am J Med 1997;103:498-503.

9. Barton JC, McDonnell SE, Adams PC, Brissot P, Powell LW, Edwards CQ, Cook JD, Kowdley KV, and the Hemochromatosis Management Working Group. Management of hemochromatosis. Ann Intern Med 1998;129:932-939.

10. McDonnell SM, Preston BL, Jewell SA, Barton JC, Edwards CQ, Adams PC, Yip R. A survey of 2,851 patients with hemochromatosis: symptoms and response to treatment. Am J Med 1999;106:619-624.

11. Collins FS. Genetics. An explosion of knowledge is transforming clinical practice. Geriatrics 1999;54:41-47.

12. Stephenson J. As discoveries unfold, a new urgency to bring genetic literacy to physicians. JAMA 1997;278:1225-1226.

13. Collins FS. Avoiding casualties in the genetic revolution: the urgent need to educate physicians about genetics. Acad Med 1999;74:48-49.

14. Hofman KJ, Tambor ES, Chase GA, Geller G, Faden RR, Holtzman NA. Physicians' knowledge of genetics and genetic tests. Acad Med 1993;688:625-632.

15. Hayflick SJ, Eiff MP, Carpenter L, Steinberger J. Primary care physicians' utilization and perceptions of genetic services. Genet Med 1998;1:13-21.

16. Emery J, Watson E, Rose P, Andermann A. A systemic review of the literature exploring the role of primary care in genetic services. Fam Pract 1999;16:426-445.

17. Kohli M, Schichman SA, Fink L, Zent CS. Use of HFE mutation analysis for hereditary hemochromatosis: the need for physician education in the translation of basic science to clinical practice. South Med J 2000;93:469-471.

18. Fry A, Campbell H, Gudmundsdottir H, Rush R, Porteous M, Gorman D, Cull A. GPs' views on their role in cancer genetics services and current practices. Fam Pract 1999; 16:468-474.

19. Mantel N, Haenszel W. Statistical aspects of the analysis of data from retrospective studies of disease. J Natl Caner Inst 1959;22:719-748.

20. Fisher RA. The logic of inductive inference. J R Stat Soc A 1935;98:39-54.

21. Hosmer DW, Lemeshow S. A goodness of fit test for the multiple logistic regression model. Commun Stat 1980;A10:1043-1069.

22. Acton RT, Burst NM, Casebeer L, Ferguson SM, Greene P, Laird BL, Leviton L. Knowledge, attitudes, and behaviors of Alabama's primary care physicians regarding cancer genetics. Acad Med 2000;75:850-852.

23. Tambor ES, Chase GA, Faden RR, Geller G, Hofman KJ, Holtzman NA. Improving response rates through incentive and follow-up: the effect on a survey of physicians' knowledge of genetics. Am J Public Health 1993;1599-1603:

24. Wilson WG. Where is the "gene" in the generalist literature? Acad Med 1998;73:931932.

25. Davis DA, Thompson MA, Oxman AD, Haynes RB. Changing physician performance: a systemic review of continuing medical education strategies. JAMA 1995; 274:700-705.

26. Ulrich CM, Kristal AR, White E, Hunt JR, Durfy SJ, Potter JD. Genetic testing for cancer risk: a population survey on attitudes and intention. Community Genet 1998; 1:213-222.

27. Mouchawar J, Klein C, Mullineaux L. Colorado family physicians' knowledge of hereditary breast cancer and related practice. J Cancer Educ 2001;16:33-37.

28. Todara HMS, Skinner CS, Gidday L, Ivanovich JL, Rawl S, Whelan AJ. Perceptions of genetic risk assessment and education among first-degree relatives of colorectal cancer patients and implications for physicians. Fam Pract 2001;18:367-372. 